\title{
Development of a Dual-Luciferase Reporter System for In Vivo Visualization of MicroRNA Biogenesis and Posttranscriptional Regulation
}

\author{
Ji Young Lee ${ }^{1,2}$, Soonhag Kim²,3, Do Won Hwang ${ }^{1,2}$, Jae Min Jeong ${ }^{2}$, June-Key Chung ${ }^{2}$, Myung Chul Lee ${ }^{2}$, and \\ Dong Soo Lee ${ }^{1,2}$ \\ ${ }^{1}$ Interdisciplinary Program in Brain Science, Seoul National University, Seoul, Korea; ${ }^{2}$ Department of Nuclear Medicine, Seoul National \\ University College of Medicine, Seoul, Korea; and ${ }^{3}$ Medical Research Center, Seoul National University College of Medicine, \\ Seoul, Korea
}

MicroRNAs (miRNAs) function in mammalian cells via translational repression or messenger RNA (mRNA) cleavage of target genes by base-pairing with $3^{\prime}$ untranslated regions (UTRs) of target mRNAs. Although miRNAs are involved in cell differentiation or organ development, posttranscritptional regulation of miRNA is not well understood. Here, we developed a dual-luciferase reporter system for monitoring in vivo endogenous transcription of primary miRNA (pri-miRNA) and also the mature miRNA activity simultaneously. Methods: miR23P639/Fluc plasmid carrying firefly luciferase (Fluc) under the control of miR-23a promoter was used to monitor the transcriptional level of miR-23a, and a cytomegalovirus (CMV)/Gluc/3xPT_mir23 recombinant containing 3 copies of the target sequence of miR-23a in the $3^{\prime}$ UTR of Gaussia luciferase (Gluc) before the poly(A) tail was used to monitor the targeting activity of mature miR-23a. This dual-luciferase reporter system transfected to the same population of cells was used to monitor the increased transcriptional level of the pri-miR23a reflected in the Fluc activity and the decreased Gluc activity affected by mature miR-23a action. Fluc and Gluc activities were also imaged in vivo using the respective substrates in grafted cells in the same nude mice using an in vivo bioluminescence imager. Results: In HeLa cells and undifferentiated P19 cells, the increased Fluc activity representingthe primary miR-23a transcript level reflected the resultant increase in repression of Gluc activity representing mature miR-23a activity. However, 293 cells showed Gluc activity was not repressed as much as Fluc activity was increased, suggesting a block in the posttranscriptional processing of miR-23a transcript in 293 cells. The miR-23a expression in P19 cells before and after neuronal differentiation with retinoic acid treatment showed an increase in Fluc activity and a concomitant decrease in Gluc activity in vitro. HeLa, 293 cells and undifferentiated P19 cells grafted to the nude mice showed exactly the same pattern of luciferase activities in vivo and in vitro.

Received Apr. 4, 2007; revision accepted Oct. 29, 2007.

For correspondence or reprints contact either of the following:

Dong Soo Lee, MD, PhD, Department of Nuclear Medicine, Seoul National University College of Medicine, 28 Yeongeon-dong, Jongno-gu, Seoul 110744, Korea.

E-mail: dsl@plaza.snu.ac.kr

Soonhag Kim, PhD, Department of Nuclear Medicine, Seoul National University College of Medicine, 28 Yeongeon-dong, Jongno-gu, Seoul 110744, Korea.

E-mail: kimsoonhag@empal.com

COPYRIGHT @ 2008 by the Society of Nuclear Medicine, Inc.
Conclusion: We developed a dual-luciferase reporter system to monitor expression and posttranscriptional regulation of a miR$23 a$ in cells in vitro and in vivo. This dual-luciferase reporter system is intended to be used to monitor the expression and regulation of miRNAs noninvasively, especially to understand the differentiation of grafted cells in vivo.

Key Words: optical imaging; microRNA; firefly luciferase; Gaussia luciferase; P19 neurogenesis; dual-luciferase reporter system

J Nucl Med 2008; 49:285-294

DOI: 10.2967/jnumed.107.042507

M icroRNAs, or miRNAs, are small noncoding RNA molecules of 17- to 24-nucleotide (nt) single-stranded RNA that are ubiquitously expressed both in plants and animals. Recently, miRNAs were found to regulate gene expression at the posttranscriptional level $(1,2)$ and act as key regulators in diverse regulatory pathways, including early development (3), cell differentiation (4), fat metabolism (5), cell proliferation, and cell death $(5,6)$. Biologic functions of miRNAs in normal, developing, and malignant cells are about to be understood by recent studies $(7,8)$. However, miRNA's main function on posttranscriptional regulation-such as messenger RNA (mRNA) degradation or translational repression of its target genes-is not as well understood.

In animals, mature miRNAs are generated via sequential processing involving translocation of partially processed precursor miRNA (pre-miRNA) from the nucleus to the cytoplasm. First, miRNAs are expressed as primary miRNAs (pri-miRNAs), which have a hairpin structure (9). Pri-miRNAs are cleaved by the double-stranded RNA (dsRNA)-specific ribonuclease, Drosha, which excises a stem-loop pre-miRNA of $\sim 70 \mathrm{nt}$ from pri-miRNAs in the nucleus (10). Pre-miRNAs are then exported by Exportin-5 (Exp5) to the cytoplasm $(11,12)$, where Dicer then cleaves pre-miRNA to approximately the 19-base-pair (bp) length of single-stranded mature miRNA (13). This single-stranded mature miRNA is then associated with the RISC (RNA-induced silencing 
complex) (13) and base-pairs with its target mRNAs, which are perfectly or imperfectly matched with the relevant miRNA sequences (1). This results in the cleavage of target mRNAs or the inhibition of their translation $(1,2)$.

Although the knowledge of miRNA biogenesis is inadequate to understand the meaning of processing of miRNA in the cells $(7,8)$, knowledge of the posttranscriptional regulation of miRNA transcripts in various cell types would give us a better understanding of the role of miRNAs in cellular regulatory pathways. Until now, Northern blotting, microarrays, and cell-specific RNA cloning approaches have been widely used to detect levels of endogenous miRNAs at various steps of posttranscriptional regulation of miRNAs (14-16). However, these techniques are time-consuming and laborious and cannot be performed repeatedly on the same subjects. Several studies adopted green fluorescent protein (GFP) or $\beta$-galactosidase as reporters to visualize miRNA expression for ex vivo imaging $(17,18)$. However, in vivo imaging using these reporter genes still suffers from invasiveness, nonrepeatabilty, or high background fluorescence as the bottleneck. Recent remarkable advances in molecular imaging techniques using bioluminescent reporter genes have provided the capability of noninvasive repeated quantitative imaging of tumor and stem cells in living animals $(19,20)$.

Here, we propose an in vivo noninvasive imaging system that can monitor endogenous miRNA biogenesis and its posttranscriptional processing by using 2 different bioluminescent proteins, firefly luciferase (Fluc) and Gaussia luciferase (Gluc). Fluc was adopted from the light-emitting organ of the firefly Photinus pyralis (21), whereas Gluc was recently cloned from the marine copepod Gaussia princes (22). These bioluminescent reporter proteins can produce light using the respective substrates. Fluc emits light with a peak at $562 \mathrm{~nm}$ during the oxidation of its substrate, beetle D-luciferin (benzothiazole) $(23,24)$, whereas Gluc uses coelenterazine as a substrate and emits light at the 480-nm peak with a broad spectrum extending to $600 \mathrm{~nm}$ (22). Fluc gene that was located downstream to a miRNA promoter was a gift from Dr. V. Narry Kim, and Gaussia luciferase gene having a miRNA target sequence was designed to reside upstream of its poly(A) tail. The transcription of miRNA is temporally controlled by its own promoter in the former vector and, thus, Fluc activity controlled by the same $5^{\prime}$ terminal regulatory promoter of miRNAs could represent the concentration of pri-miRNA at the transcription level $(18,25)$. A constitutively expressing Gluc bearing complementary sequences to a miRNA in its $3^{\prime}$ untranslated region (UTR) is repressed in cells expressing the specific miRNA and could represent the activity of the mature form of this miRNA. These different expressions of luciferases allow both endogenous pri-miRNA and mature miRNA to be monitored simultaneously in the same population of cells, as these 2 luciferases use different substrates and have no cross-activity between these enzymes. Now, by imaging 2 luciferase activities in the same subjects, it is possible to minimize inherent variability that happens in classical biodistribution studies affected by individual dif- ferences of the animal subjects and the cultured cells and their efficiencies of transgene transfection and others.

For the development of a dual-luciferase system to follow miRNA biogenesis and its posttranscriptional regulation, miR-23a was selected (25) and the $5^{\prime}$ regulatory sequence controlling the miR23a $\sim 27 \mathrm{a} \sim 24-2$ gene cluster was used to determine whether the degree of miR-23a transcription is cell-type dependent or differentiation-stage specific. The complementary nucleotide sequences of mature miR-23a were used to detect the endogenous level of mature miR-23a. Using this dual-luciferase system we could evaluate the expression patterns of miR-23a in specific cell types and various stages of differentiation and follow or visualize intracellular molecular events of miR-23a biogenesis in a noninvasive manner of bioluminescent molecular imaging.

\section{MATERIALS AND METHODS}

\section{Plasmid Construction of MiR23P639/Fluc and CMV/Gluc/3xPT_mir23}

miR23P639/Fluc was kindly donated by Dr. V. Narry Kim (25). Nucleotides -603 to +36 upstream of the gene cluster of primiR23a 27a 24-2 were fused upstream to the firefly luciferase (Fig. 1A). This Fluc reporter system was used to monitor the expression of pri-miR-23a.

We constructed a Gluc reporter vector (cytomegalovirus (CMV)/ Gluc/3xPT_mir23) bearing 3 copies of a perfect complementary miR-23a target sequence (3xPT_mir23), which was fused immediately after the Gluc stop codon. The nucleotide sequences of the precursor has-mir-23a and mature has-mir-23a were obtained from the miRNA registry (26), and we constructed 3 copies of a perfect complementary target sequence of mature miR-23a (3xPT_mir23a) (Fig. 1B) by annealing the following oligonucleotides: 5 '-tcgagaatctagtGGAAATCCCTGGCAATGTGATtagtaGGAAATCCCTGGCAATGTGATtagtaGGAAATCCCTGGCAATGTGATt-3', 5' -ctagaATCA CATTGCCAGGGATTTCCtactaATCACATTGCCAGGGATTTCCtactaATCACAT TGCCAGGGATTTCCactagattc-3' . The doublestranded inverse sequences of 3xPT_mir23a (3xPT_mir23_inv) (Fig. 2A) were induced by annealing the following single-stranded oligonucleotides: 5' -tcgagTAGTGTAACGGTCCCTAAAGGatgatTAGTGTAACGGTCCCTAAAGGatgat TAGTGTAACGGTCCCTAAAGGtgatctaat-3', 5' -ctagattagatcaCCTTTAGGGACCG TTACACTAatcatCCTTTAGGGACCGTTACACTAatcatCCTTTAGGGACCGTTACACTAc-3'. The double-stranded oligonucleotide was subcloned into the $X h o 1$ and $X b a 1$ site of CMV/Gluc to create CMV/Gluc/3xPT_mir23 and CMV/Gluc/3xPT_mir23_inv. Three copies of perfect targets before the poly(A) tail enhanced the response of CMV/Gluc to the repressive activity of mature miR-23a.

To construct Fluc vector to overexpress pri-miR23a, the CMV promoter from CMV/Fluc vector was inserted into the $K p n 1$ and Xba1 sites of pGL3_basic to create pGL3/CMV. Then, we constructed the pri-miR23a sequence by annealing the following oligonucleotides: 5'-tcgagcecctcaccectgtgccacGGCCGGCTGGGGTTCCTGGGGATGGGATTTGCTTCCTGTCACAAATCACATTGCCAGGGATTTCCAACCGACCctgagctctgccaccgaggac-3', 5' -tcgagtcctcggtggcagagctcagGGTCGGTTGGAAATCCCTGGCAATGTGATTTGTGAC AGGAAGCAAATCCCATCCCCAGGAACCCCAGCCGGCCgtggcacaggggtgagggggc- 3 '. The resulting vector, pGL3/CMV, was subsequently digested with $K p n 1$ and $X b a 1$ and the pri-miR23a sequence was ligated into the Kpn 1 and $X b a 1$ sites of pGL3/CMV 


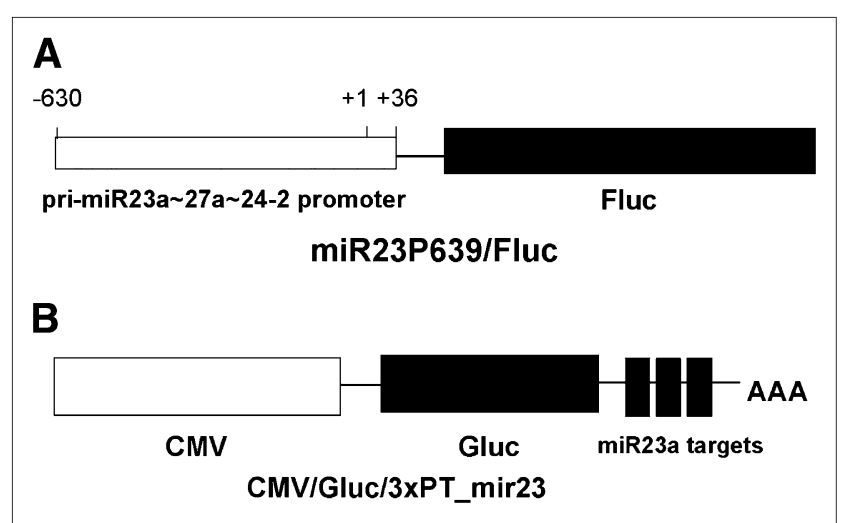

FIGURE 1. Reporter constructs to monitor primary transcript and mature miRNA activities. (A) miR23P639/Fluc. Segment ranging from 603 to $36 \mathrm{bp}$ upstream to the transcription initiation site of miR23a 27a 24-2 gene clusters was inserted into pGL3_basic (Promega) containing a promoterless Fluc gene. (B) CMV/Gluc/3xPT_mir23. CMV/Gluc carries 3 copies of target sequence perfectly complementary to miR-23a (3xPT_mir23) in its $3^{\prime}$ UTR.

to create CMV/pri_miR23a/Fluc. This vector was used to check the efficiency and expression/maturation of Gluc vector activity of exogenous pri-miR-23a transfected to 293 cells to validate the CMV/pri_miR23a/Fluc vector.

\section{Cell Culture of HeLa, 293, and Undifferentiated/ Differentiated P19 Cells}

HeLa cells (an adenocarcinoma cell line) were cultured routinely in RPMI medium (Jeil Biotechservices Inc.) containing 10\% fetal bovine serum (FBS; Cellgro) and 1\% Antibiotics-antimycotics (Cellgro). HEK 293 cells (human embryonic kidney cell line) were maintained in Dulbecco's modified Eagle medium (DMEM; Gibco) containing 10\% FBS and 1\% Antibiotics-antimycotics. P19 cells (a mouse embryonic carcinoma cell line) were purchased from the American Type Culture Collection. For maintenance purposes, cells were grown at $37^{\circ} \mathrm{C}$ in $\alpha$-MEM (Gibco) supplemented with $2.5 \%$ FBS, $7.5 \%$ bovine calf serum (Gibco), and $1 \%$ Antibiotics-antimycotic in a $5 \% \mathrm{CO}_{2}$-humidified chamber (27). To induce neuronal differentiation, cells were then cultured under serum-free conditions in DMEM/F12 (1:1) media (Gibco) supplemented with insulin, transferrin, and selenium (Gibco) and $5 \times 10^{-7} \mathrm{M}$ all-trans-retinoic acid (RA; Sigma) according to the method described by Ying et al. (28). After $2 \mathrm{~d}$, RA was withdrawn from the medium and cells were cultured further under serum-free conditions.

\section{Transfection of MiR23P639/Fluc and CMV/Gluc/ 3xPT_mir23}

HeLa or 293 cells were seeded, at $1 \times 10^{5}$ cells per well in a 24 well plate $24 \mathrm{~h}$ before transfection. Transfection was performed with LipofectAMINE Plus reagent (Invitrogen), using $0.6 \mu \mathrm{g}$ of DNA, $3 \mu \mathrm{L}$ of Plus reagent, and $1.5 \mu \mathrm{L}$ of LipofectAMINE per well. The functional expression of each complementary DNA (cDNA) was analyzed $48 \mathrm{~h}$ after transfection. All transfections were performed in triplicate.

To validate CMV/Gluc/3xPT_mir23 activity representing mature miR-23a activity, 2 validation sets of experiment were designed. First, CMV/Gluc/3xPT_mir23 was transfected to HeLa cells, which produce endogenously mature miR-23a. CMV/Gluc/3xPT_mir23_ inv was used as a negative control (Fig. 2A). CMV/Gluc was used as an internal control. Second, CMV/Gluc/3xPT_mir23 was cotransfected with CMV/pri_miR23a/Fluc to 293 cells, which do not produce mature miR-23a as themselves (Table 1). CMV/Fluc was used as an internal control for CMV/pri_miR23a/Fluc and CMV/ Gluc was for CMV/Gluc/3xPT_mir23.

To examine the level of primary miR-23a transcript and the activity of mature miR-23a simultaneously, 3 sets of transfection combination were designed (Table 2). In an "Experiment" set, miR23P639/Fluc and CMV/Gluc/3xPT_mir23 were cotransfected into HeLa, 293 and P19 cells to monitor pri-miR23a transcription and mature miR-23a activity simultaneously (Table 2, second column). In a "Control for Fluc" set, CMV/Gluc/3xPT_mir23 was cotransfected along with promoter-less pGL3_basic encoding Fluc as an internal control for P639 promoter activities (Table 2, first column). In a third "Control for Gluc" set, miR23P639/Fluc was cotransfected with CMV/Gluc as another internal control, which did not contain miR-23a target sequences (Table 2, third column). After transfection into cells, both Fluc and Gluc activities were measured using D-luciferin and coelenterazine, and relative levels of luciferase were measured by normalization to the respective internal controls.

\section{Luciferase Assay for Fluc and Gluc Activities}

Forty-eight hours after transfection, cells were washed with phosphate-buffered saline (PBS, $\mathrm{pH}$ 7.4) and treated with lysis buffer for luciferase assays. Luciferase activities were measured according to the manufacturer's instructions (Dual-luciferase assay system; Promega). Data are presented as mean \pm SD calculated from triplicate wells. Significant differences between samples were assessed using the Student $t$ test. $P$ values $<0.05$ were considered statistically significant.

\section{Reverse Transcription Polymerase Chain Reaction (RT-PCR) of Stem Cell/Differentiation Markers and Pri-miR-23a}

Total RNA was isolated from cultured cells using Trizol reagent (Invitrogen). Reverse transcription for synthesizing the firststrand cDNA was performed using a random-hexamer primer and SuperScript II reverse transcriptase (Invitrogen), according to the manufacturer's instructions, and used as a template for PCR amplification. The sequences of the primers used for PCR amplification were 5'-CCCAAGAACCAACAAGATGAA-3' (MAP2 forward) and 5'-AATCAAGGCAAGACATAGCGA-3' (MAP2 reverse), 5'-GGCGTTCTCTTTGGAAAGGTGTTC-3' (Oct4 forward) and 5'-CTCGAACCACATCCTTCTCT-3' (Oct4 reverse), 5'-TGACGGGGTCACCCAACTGTGCCCATCTA-3' ( $\beta$-actin forward) and 5'-CTAGAAGCATTTGCGGTGGACGATGGAGGG-3' $(\beta$-actin reverse). PCR amplifications of MAP2, Oct 4 , and $\beta$-actin cDNA were performed using i-Taq DNA polymerase (iNtRON). PCR products were loaded on agarose gels containing ethidium bromide, and bands were revealed under ultraviolet light. For primiR-23a $\sim 27 \mathrm{a} \sim 24-2$, first-strand cDNA synthesis was performed using a random-hexamer primer, and 5'-CCCTGTTCCTGCTGAACTGAGCCAGTGTAC-3' (reverse) and 5'-CGCCCGGTGCCCCCCTCACCCCTGTGCCAC-3' (forward) were used for PCR amplification.

\section{Quantitative RT-PCR (qRT-PCR) of Mature miRNA23a}

Small RNA was isolated from cultured cells using mirVana miRNA isolation kits (Ambion). qRT-PCR was performed with mirVanaTM qRT-PCR miRNA detection kits (Ambion) using a has-miR-23a primer set (Ambion) according to the manufacturer's 


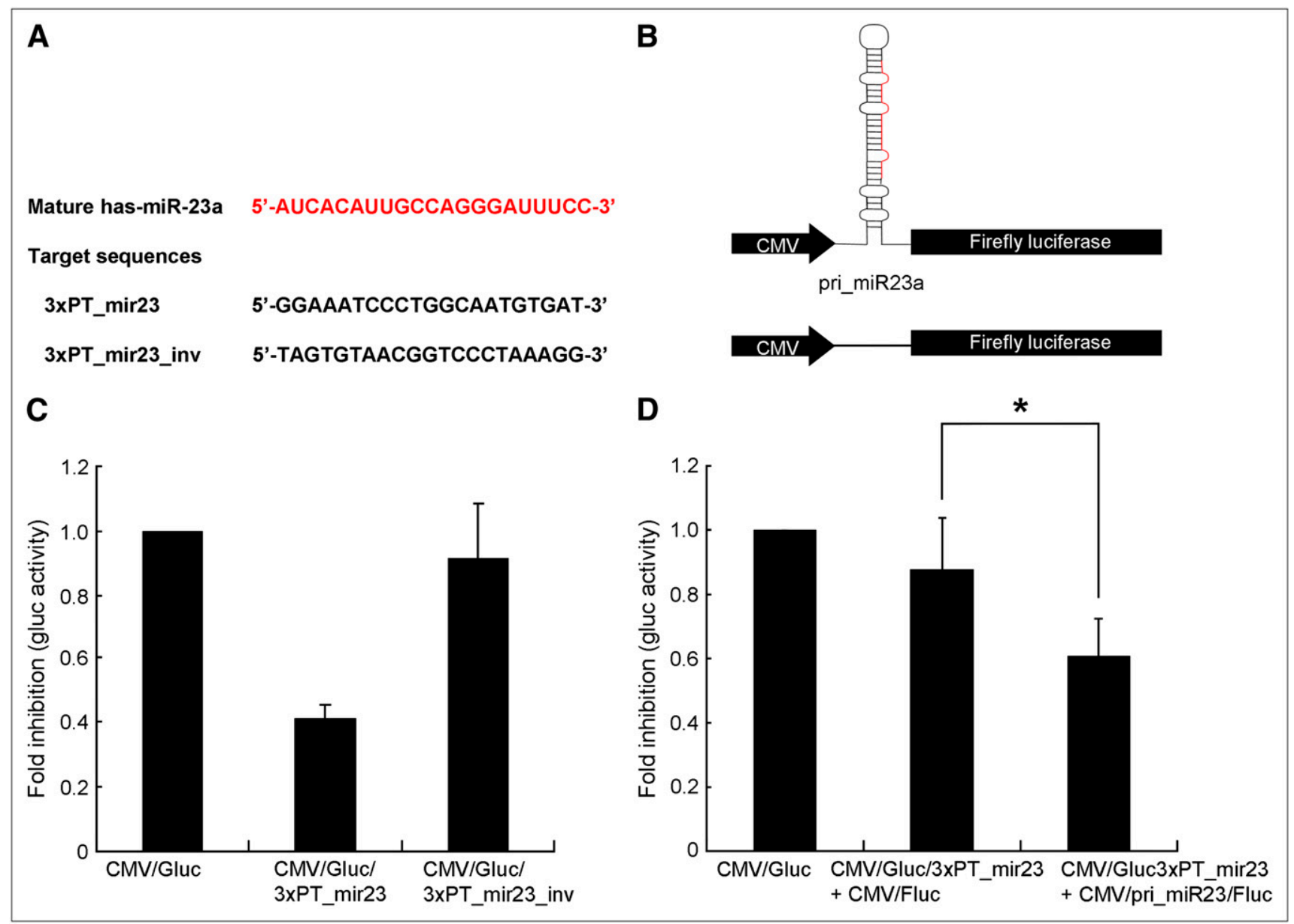

FIGURE 2. Validation of CMV/Gluc/3xPT_mir23 construct to represent mature miR-23a activity. (A) Mature miR-23a sequence and its relevant target sequence, which were inserted into the 3' UTR of Gluc reporter gene. Two targets of 3xPT_mir23 sequences were constructed; one was a perfect complement and the other was an inverted sequence and was used as negative control. (B) Constructs of CMV/pri-miR-23a/Fluc and CMV/Fluc used as exogenous source and its negative control of pri-miR-23a. (C) CMV/ Gluc/3xPT_mir23 and CMV/Gluc/3xPT_mir23_inv were transfected into HeLa cells. Endogenous mature miR-23a in HeLa cells repressed Gluc activity of transfected CMV/Gluc/3xPT_mir23 construct but did not repress that of transfected CMV/Gluc/ 3xPT_mir23_inv construct. (D) CMV/pri-miR-23a/Fluc and CMV/Gluc/3xPT_mir23 were transfected into 293 cells with their respective controls of CMV/Fluc and CMV/Gluc. Exogenously expressed pri-miR-23a should have been processed to mature miR23a and, thus, repressed Gluc activity partially in 293 cells cotransfected with CMV/pri-miR23a/Fluc and CMV/Gluc/3xPT_mir23. Cotransfected CMV/Fluc, which does not express pri-miR-23a, did not repress Gluc activity of 293 cells transfected with CMV/ Gluc/3xPT_mir23. * $P<0.001$.

instructions. To normalize experimental samples for RNA content, the U6 snRNA primer set (Ambion) was used as a control.

\section{Grafting of Cells with MiR23P639/Fluc and CMV/Gluc/ 3xPT_mir23 and In Vivo Visualization of Expression and Activity of miR-23a in Nude Mice}

The vectors miR23P639/Fluc, CMV/Gluc/3xPT_mir23, pGL3_ basic, and CMV/Gluc, were transiently transfected into 293, HeLa, or P19 cells for in vivo imaging of miR-23a biogenesis. After $24 \mathrm{~h}$ of transfection, cells were counted and resuspended in $100 \mu \mathrm{L}$ PBS $\left(1 \times 10^{7}\right.$ cells $/ 100 \mu \mathrm{L}$ PBS $)$. These cells were implanted subcutaneously into the flanks of male BALB/c nude mice (6-wk-old, weighing 25-27 g). The cells cotransfected with pGL/basic and CMV/Gluc vectors were implanted into the left sides as the control, and the cells cotransfected with miR23P639/Fluc and CMV/ Gluc/3xPT_mir23 were implanted into the right sides. All mice were anesthetized in an IVIS imaging chamber (Xenogen) equipped with a small holder connected to an isoflurane/oxygen main tank, and anesthetic gas was administered with $2.5 \%$ isoflurane gas in oxygen at a flow of $1.5 \mathrm{~L} / \mathrm{min}$.

When imaging Gluc and Fluc in the same mouse, mice were tail-vein injected with $100 \mu \mathrm{g}$ of coelenterazine, and Gluc images were acquired $2 \mathrm{~min}$ later. Ten minutes after coelenterazine injection, $4 \mathrm{mg}$ (150 mg/kg body weight) of D-luciferin was injected again intraperitoneally and Fluc images were acquired 10 min after D-luciferine injection. The whole-body images for Gluc activity and Fluc activity were acquired for 1 and $5 \mathrm{~min}$, respectively.

\section{RESULTS}

Validation of CMV/Gluc/3xPT_mir23 Activity to Represent Mature Endogenous or Exogenous miR-23a

Gluc reporter vector containing miR-23a target sequences was tested whether it was repressed by endogenous 
miR-23a in HeLa cells or by exogenous miR-23a in CMV/ pri_miR23a/Fluc transfected 293 cells. The repression of Gluc activity from transfected CMV/Gluc/3xPT_mir23 in HeLa cells was considered to represent mature miR-23a biogenesis. To exogenously overexpress and monitor mature miR-23a activity, we cotransfected CMV/Gluc/ 3xPT_mir23 and CMV/pri_miR23a/Fluc into 293 cells and examined the repression of Gluc activity by miR23a's cleavage of Gluc mRNA of CMV/Gluc/3xPT_mir23 vector.

In an endogenous model of miR23a biogenesis in HeLa cells, Gluc activity from CMV/Gluc/3xPT_mir23 was repressed by existing miR23a but Gluc activity from CMV/ Gluc/3xPT_mir23_inv used as a negative control was barely changed (Fig. 2C). In an exogenous model of 293 cells, cotransfection of CMV/Gluc/3xPT_mir23 and CMV/ pri_miR23a/Fluc significantly repressed Gluc activity due to overexpressed mature miR23a from CMV/pri_miR23a/ Fluc plus endogenous mature miR23a, while Gluc expression from cotransfection of CMV/Gluc/3xPT_mir23 and $\mathrm{CMV} /$ Fluc was just decreased as much as mature miR23a endogenously expressed in 293 cells (Fig. 2D).

\section{Detection of Endogenous Expression of Pri-miR23a and Mature miR-23a Activity In Vitro by Fluc and Gluc Activities}

Fluc activity of miR23P639/Fluc transfected cells represented high levels of pri-miR23a expression under the control of miR-23a promoter $(-603$ to +36$)$. Gluc activity of CMV/Gluc/3xPT_mir23 transfected cells represented mature miR-23a activity as shown earlier.

After cotransfection of miR23P639/Fluc and CMV/Gluc/ 3xPT_mir23 into HeLa and 293 cells, Fluc activity driven by miR-23a promoter increased by 17 -fold and 23 -fold versus promoter-less Fluc expressions, respectively (Fig. $3 \mathrm{~A})$. In the same population of cotransfected cells, Gluc activity resulting from the CMV/Gluc/3xPT_mir23 construct was repressed about 3-fold in response to endogenous mature miR-23a in HeLa cells; on the other hand, Gluc repression was barely observed in 293 cells (Fig. 3B). The lack of repression of Gluc activity observed in 293 cells represented a low amount of endogenous mature miR-23a. This lack of a correlation between pri-miR23a expression and the mature miR-23a level in 293 cells implied that there was a partial block in the processing of pri-miR23a to mature miR-23a in these 293 cells.

In RT-PCR with primers specific for pri-miR23a to compare the primary transcript level, the quantity of primiR23a was greater in 293 cells than that in HeLa cells (Fig. 4A); however, in a real-time RT-PCR to quantify endogenous mature miR-23a, the quantity of mature miR23a was greater in HeLa cells than that in 293 cells (Fig. 4B). Thus, Fluc and Gluc activities and their match or mismatch of relative quantities in HeLa and 293 cells concurred with the quantities of primary transcript and mature form of miR-23a on RT-PCR and qRT-PCR.

\section{Changes of Functional Expression and Processing of miR-23a During P19 Neurogenesis In Vitro}

To observe the functionally variable expression of miR23a during neurogenesis, in a mouse embryonic carcinoma cell line (P19 cells), that differentiates into neuronal cells when treated with RA, we investigated the pattern of expression and posttranscriptional processing of miR-23a (27). Neuronal differentiation was induced by stimulation with RA under serum-free conditions (Fig. 5A) and was confirmed by the disappearance of the Oct4 (stem cell) marker and the appearance of the MAP2 (neuron) marker (Fig. 5B).

miR23P639/Fluc and CMV/Gluc/3xPT_mir23 were cotransfected into P19 cells in undifferentiated states and after 3 and $9 \mathrm{~d}$ of differentiation ( 1 and $7 \mathrm{~d}$ of RA withdrawal after $2 \mathrm{~d}$ of treatment). Fluc activity, which was 10 -fold higher in undifferentiated cells compared with that of the negative control, decreased with neuronal differentiation (Fig. 6A). This decrease represented the decreased expression of pri-miR-23a in differentiated neuronal cells. Gluc activity, which was repressed by $20 \%$ compared with that of CMV/Gluc activity in undifferentiated P19 cells, increased because of the decreased amount of mature miR23a (Fig. 6B).

The amount of primary transcript of pri-miR23a decreased on RT-PCR in differentiated P19 cells (Fig. 6C), and the amount of mature miR-23a decreased concordantly on qRT-PCR compared with that of undifferentiated cells (Fig. 6D). These data showed that miR-23a expression and its mature form decreased during P19 neurogenesis after RA stimulation.

TABLE 2

Experimental Scheme Using 3 Sets of pri-miR23a Expression and Mature miR-23a Activity

\begin{tabular}{ccc}
\hline Control for Fluc & Experiment & Control for Gluc \\
\hline pGL3_basic + CMV/Gluc/3xPT_mir23 & miR23P639/Fluc + CMV/Gluc/3xPT_mir23 & miR23P639/Fluc + CMV/Gluc \\
\hline
\end{tabular}


FIGURE 3. Enhancement of Fluc representing endogenous expression of miR-23a and repression of Gluc representing mature miR-23a activity in HeLa and 293 cells. (A) Compared with pGL_basic activity, miR23P639/Fluc transfection revealed 10 -fold increase in firefly luciferase activities in HeLa and 293 cells. Increased Fluc activities represented increased expression of endogenous pri-miR-23a in these cells. (B) Compared with CMV/Gluc transfection, CMV/Gluc/3xPT_mir23 transfection revealed decrease of Gaussia luciferase activities in HeLa cells but not in 293 cells. Repressed Gluc activities represented the presence of mature miR-23a activity and, thus, concordant expression of miR-23a in HeLa cells but discordant expression of pri-miR-23a as well as the activity of mature miR-23a in 293 cells. Data are expressed as mean \pm SD of triplicates.

\section{Visualization of Expression of Pri-miR23a and Activity of Mature miR-23a In Vivo}

The dual-luciferase system enabled in vivo visualization of HeLa $(n=2), 293(n=3)$, and undifferentiated P19 cells $(n=3)$ cotransfected with miR23P639/Fluc and CMV/Gluc/3xPT_mir23, which was also quantified by region-of-interest (ROI) analysis. Figure 7 depicts repre-

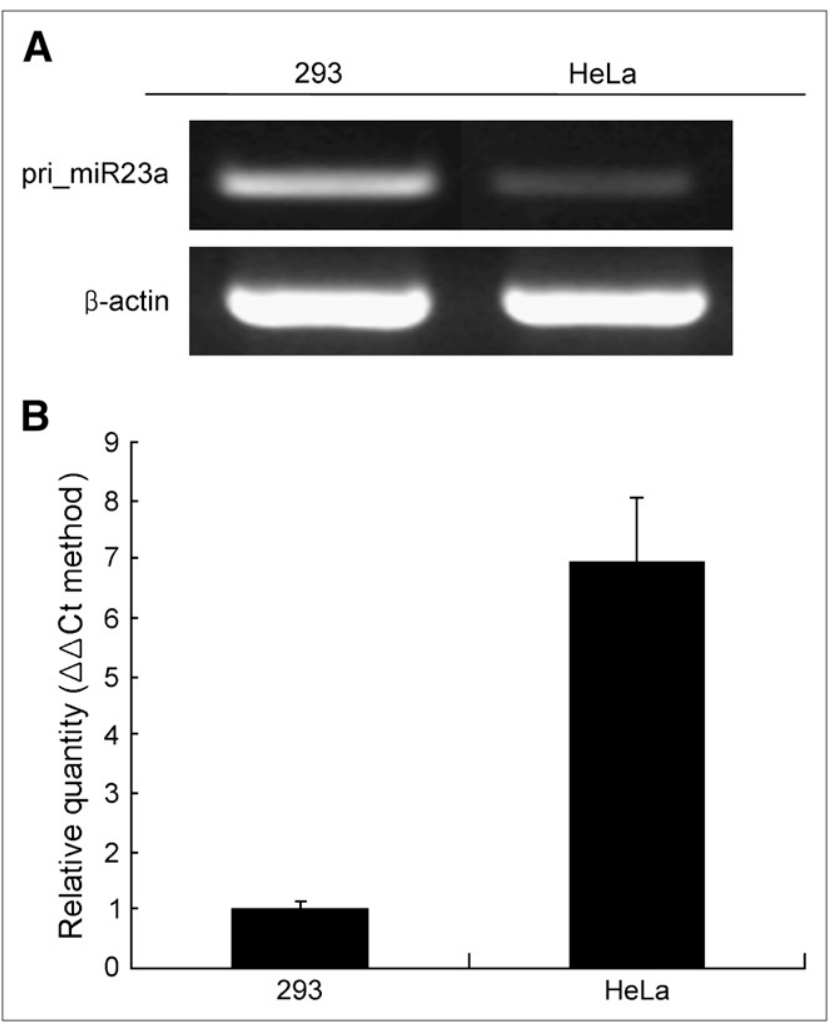

FIGURE 4. (A) Levels of endogenous pri-miR-23a were determined by RT-PCR in HeLa and 293 cells. $\beta$-Actin was used as control. (B) Relative quantity of mature miR-23a on real-time qRT-PCR in HeLa and 293 cells. Values are mean \pm SD of triplicates. U6 snRNA was used as an endogenous control. $\Delta \Delta \mathrm{Ct}=\Delta \Delta$ threshold cycle $\left(\Delta \mathrm{Ct}=\mathrm{Ct}_{\text {miRNA }}-\mathrm{Ct}_{\mathrm{U} 6 \mathrm{RNA}}\right.$, $\left.\Delta \Delta \mathrm{Ct}_{293}=\Delta \mathrm{Ct}_{293}-\Delta \mathrm{Ct}_{293}, \Delta \Delta \mathrm{Ct}_{\mathrm{HeLa}}=\Delta \mathrm{Ct}_{\mathrm{HeLa}}-\Delta \mathrm{Ct}_{293}\right)$. sentative cases, and all mice showed similar results (Supplemental Figure 1, which provides another example for 293 and P19 cells, is available online only at http:// jnm.snmjournals.org). In the mice grafted subcutaneously with HeLa, 293 and P19 cells cotransfected with miR23P639/Fluc and CMV/Gluc/3xPT_mir23, Fluc activity and Gluc activity from the control group were observed on the left side and the luciferase signal from the experimental group was observed on the right side of all animals. From both ROI and in vivo imaging analysis, Fluc activity increased substantially on the right side, showing an increase in expression of miR-23a in each grafted cell compared with the background bioluminescence emitted from the control groups. Gluc activity decreased markedly only in HeLa cells and undifferentiated P19 cells but not in 293 cells. In the in vivo model of animals, the concordant expression of primary transcript of miR-23a and the activity of mature miR-23a was documented in HeLa cells, and this was also the case with undifferentiated P19 cells. However, in 293 cells, the expression of primary transcript of miR-23a was found in vivo with barely visible mature miR-23a activity; thus, this primary transcript did not appear to be processed further to mature miR-23a, which had been observed in the previous in vitro experiment.

\section{DISCUSSION}

Animal genomes appear to contain at least 500 miRNAs that target thousands of genes that are involved in cellular differentiation and regulatory pathways (29). Some of these miRNAs are organ-specific or tissue-specific within an organ, whereas others are ubiquitous-reflecting different roles of miRNAs in different cells. Although miRNA biogenesis is not fully understood, studies of miRNA expression patterns during cellular development or in various cell types might provide meaningful insight with regard to miRNA functions. Unlike other mammalian mRNAs, miRNAs are much slower at converting from their primary transcripts into their active forms; this requires simultaneous assessment of the expression patterns of pri-miRNA and mature miRNA 

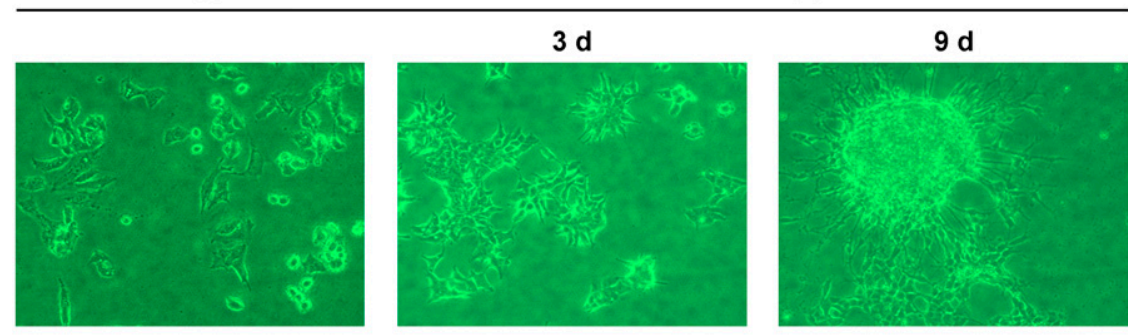

B

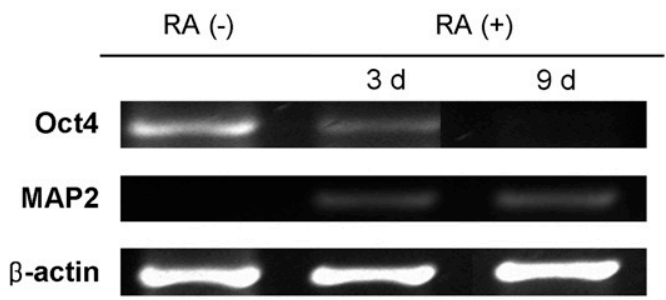

FIGURE 5. Neuronal differentiation of P19 by RA treatment. (A) Sequential phase-contrast images of P19 neurogenesis. Cells developed a neuronal morphology first by rounding up and then by producing protrusions. Finally, cells were organized into neurospherelike colonies with growing neurites. (B) RNAs were prepared from undifferentiated or differentiated P19 cells on days 3 and 9 of RA treatment and were analyzed by RT-PCR. Oct4 expression was reduced after RA treatment. RA treatment caused P19 cells to differentiate into MAP2-positive neuronal cells. activity at the cell level to understand the precise role of miRNA action in cellular development.

The dual-luciferase reporter system enabled us to monitor simultaneously the endogenous expression of pri-miRNA and the activity of mature miRNA within the same population of cells by visualization in vivo as well as in vitro. Firefly luciferase activity representing the primary miR-23a transcript level and Gaussia luciferase activity representing the mature miR-23a level were correlated in HeLa cells and undifferentiated or differentiated P19 cells-that is, increased Fluc activity of the primary transcript level was accompanied by repressed Gluc activity influenced by an

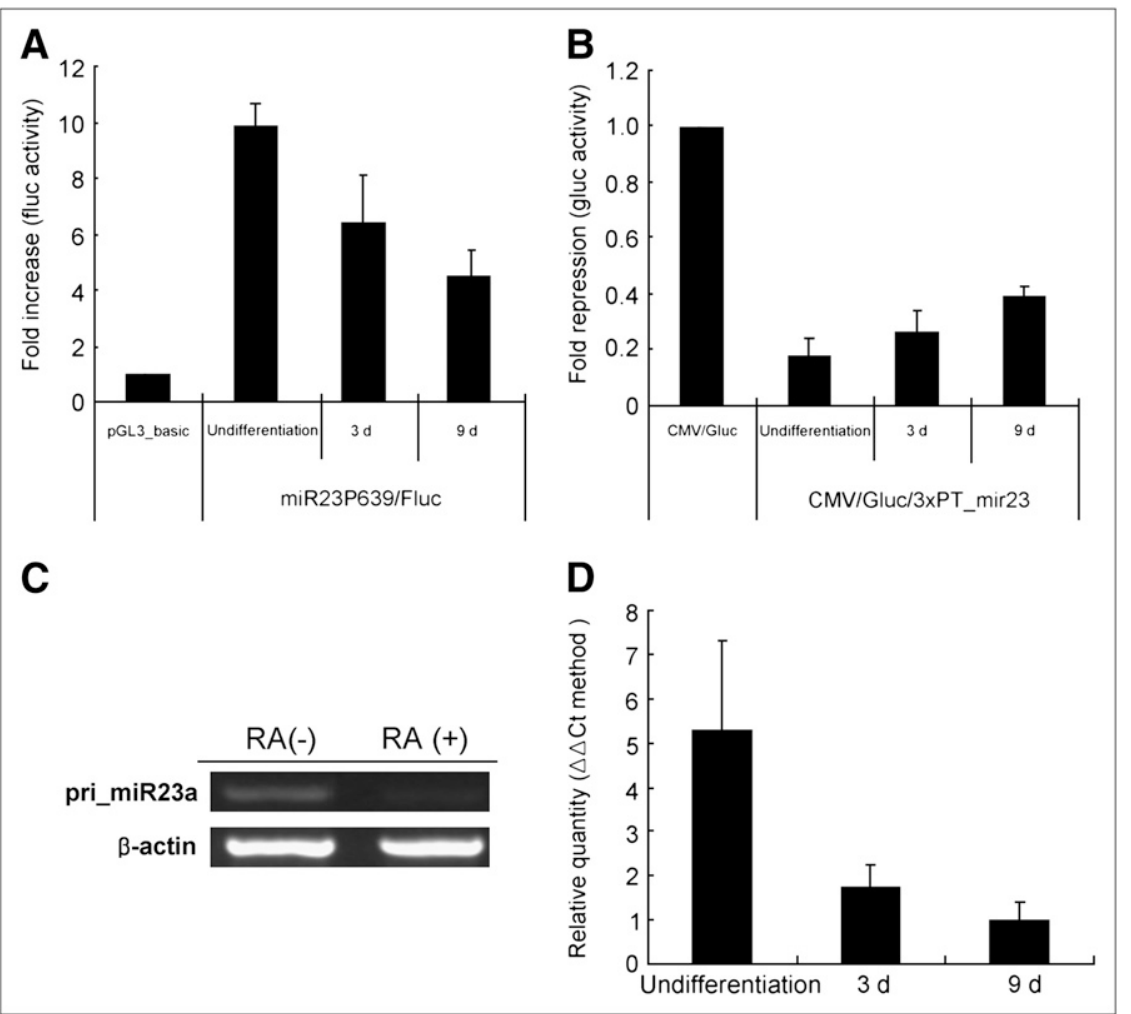

FIGURE 6. Pri-miR-23a expression and mature miR-23a activities in P19 cells during neurogenesis and confirmation of expression pattern by RT-PCR for primary miR23a and qRT-PCR for mature miR23a. (A) Compared with pGL_basic activites, miR23P639/Fluc transfection increased firefly luciferase activities in undifferentiated P19 cells, which represented enhanced expression of primary transcript of pri-miR-23a in these cells. This increased Fluc activity decreased in differentiated P19 cells on day 3 and was further reduced on day 9. (B) Compared with CMV/Gluc, CMV/Gluc/3xPT_mir23 transfection revealed decreased Gluc activity, which represented higher mature miR-23a activities. This decrease of mature miR-23a activity was improved in differentiated P19 cells on day 3 and was further improved on day 9. There was a concordance between higher expression of pri-miR-23a and high activity of mature miR-23a in undifferentiated P19 cells and decreased, but-still-present, expression of pri-miR-23a (Fluc) and decreased repression (increase) of mature miR-23a (Gluc) in differentiated P19 cells during neurogenesis. Data are expressed as means ( \pm SD) of triplicates. (C) RT-PCR of pri-miR-23a showed that expression of endogenous pri-miR-23a was reduced during P19 neurogenesis after RA treatment. $\beta$-Actin was used as control. (D) Real time qRT-PCR of mature miR-23a showed that endogenous mature miR-23a levels were reduced after P19 neurogenesis. U6 snRNA was used as control. Data are expressed as mean \pm SD of triplicates. $\Delta \Delta \mathrm{Ct}=\Delta \Delta$ threshold cycle $\left(\Delta \mathrm{Ct}=\mathrm{Ct}_{\text {miRNA }}-\mathrm{Ct}_{\mathrm{UGRNA}}, \Delta \Delta \mathrm{Ct}_{9 \mathrm{~d}}=\Delta \mathrm{Ct}_{9 \mathrm{~d}}-\Delta \mathrm{Ct}_{9 \mathrm{~d}}, \Delta \Delta \mathrm{Ct}_{3 \mathrm{~d}}=\Delta \mathrm{Ct}_{3 \mathrm{~d}}-\Delta \mathrm{Ct}_{9 \mathrm{~d}}, \Delta \Delta \mathrm{Ct}_{\mathrm{un}}=\Delta \mathrm{Ct}_{\mathrm{un}}-\Delta \mathrm{Ct}_{9 \mathrm{~d}}\right)$. 
A

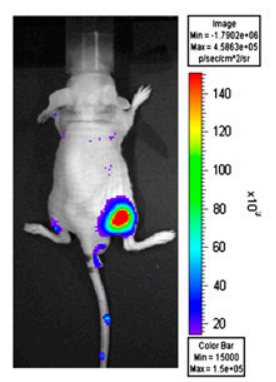

HeLa_Fluc

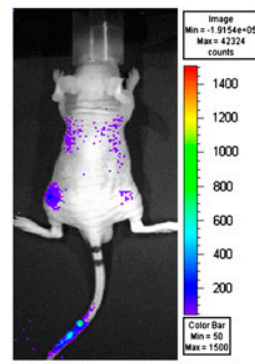

HeLa_Gluc
B
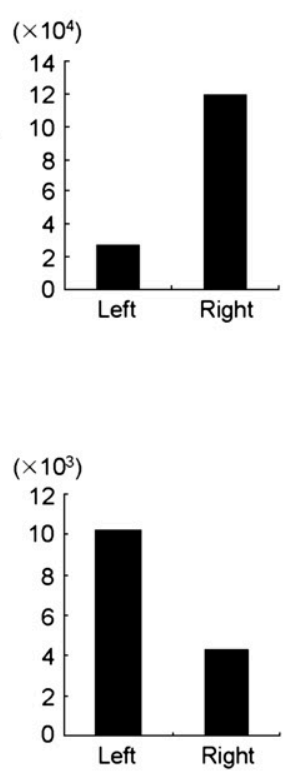
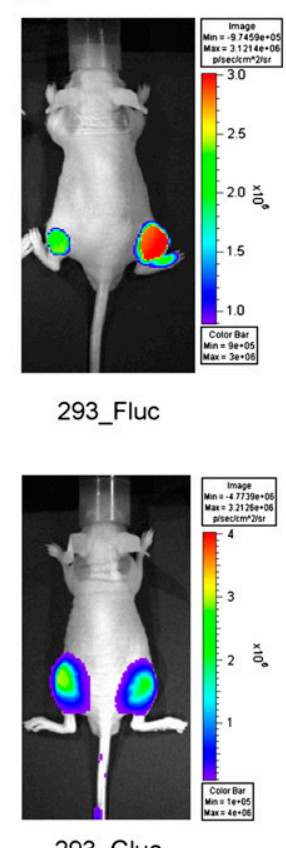

C
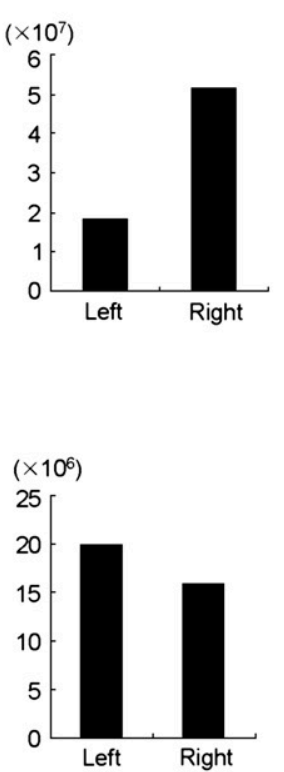
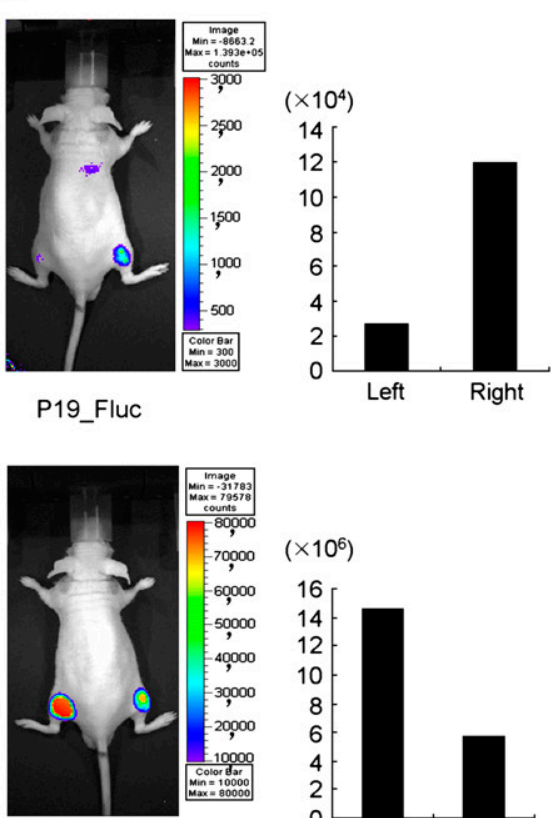

P19_Gluc

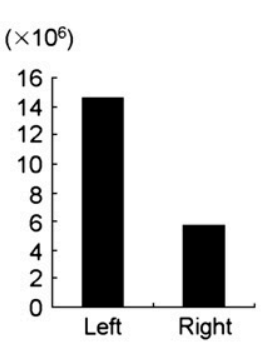

FIGURE 7. In vivo visualization of expression of pri-miR-23a transcripts and activity of mature forms of miR-23a in nude mice. HeLa cells (A), 293 cells (B), and undifferentiated P19 cells (C) were transfected with miR23P639/Fluc and CMV/Gluc/3xPT_mir23 and were grafted $\left(1 \times 10^{7}\right.$ cells) to right side of mice. Same cells transfected with pGL3_basic and CMV/Gluc were grafted to left side as controls. Quantitative data from imaging in vivo were obtained by ROI analysis and are shown next to each small-animal picture. Gluc images were acquired first with coelenterazine, and then Fluc images were then acquired with D-luciferin. When viewing Fluc images we pay attention to increased bioluminescence on right side of grafted cells expressing pri-miR-23a transcripts. When viewing Gluc images we try to find decreased bioluminescence on right side compared with luciferase activity on left-sided CMV/Gluc controls, disclosing mature miR-23a activities. Fluc activities increased in all animals on right side, and Gluc activities decreased in HeLa and P19 cells but decreased the least in 293 cells transfected with CMV/Gluc/3xPT_mir23 on right side.

increased mature miR-23a level. However, in 293 cells, although the level of primary miR-23a transcript was high, Gluc activity was not repressed by mature miR-23a as much as that of Fluc activity. The dual-luciferase reporter system could disclose this mismatch in the level of pri-miR23a and the level of the functionally active mature miR-23a in 293 cells. This disclosure indicated the capability of our system for assessment (in vitro) and visualization (in vivo) of posttranscriptional regulation of miR-23a $(7,8)$. This discrepancy in expression between endogenous pri-miR23a and mature miR-23a in 293 cells is possibly related to some unknown mechanism that regulates mature miRNA biogenesis during the Drosha or Dicer processing steps (30). The primary miR-23a was produced at a higher level than the resultant mature miR-23a, indicating a delay of Dicer processing, holding back the release of premiR23a from nucleus into cytoplasm, blocking Drosha function, or a fast turnover of mature miR-23a.

We also found that the expression of endogenous miR-23a decreased during P19 neurogenesis using this dual-luciferase reporter system. Gradual reductions in pri-miR23a and accompanying reduced activity of mature miR-23a were observed, during P19 neurogenesis, by determining decreased Fluc activity and increased Gluc activity. In undif- ferentiated P19 cells, the relative functional levels of mature miR-23a were higher than those in 293 cells and HeLa cells, and during differentiation this activity decreased and Gluc activity increased twice. And this decrease in mature miR23a activity was closely associated with the decrease in the transcription of pri-miR-23a represented by the decrease of Fluc activity during neuronal differentiation. These results imply that miR-23a plays a role in neuronal differentiation. However, miR-23a expression and posttranscriptional processing is not specific for neurons. During neurogenesis, miR-9, miR-142a, miR-124b, miR-135, miR-153, miR-183, and miR-219 are reported to express exclusively in neurons, and miR-9*, miR-125a, miR-125b, miR-128, miR-132, miR137, and miR-139 are known to be highly enriched (31). The relative roles of these miRNAs during neuronal differentiation or neurogenesis might be elucidated using our methods, whereas the individual and concerted action of these miRNAs will be proven to play roles in neurogenesis. Specific or nonspecific roles of miR-23a are of interest during neurogenesis.

Furthermore, the observed differential expression or posttranscriptional regulation demonstrates that the expression patterns of pri-miR23a and mature miR-23a are associated with the maintenance of tissue identity in cell lines as their expression patterns are uniquely significant in cancer cells 
(8). The synthetic CMV/Gluc/3xPT_mir23 construct might indirectly demonstrate one of the miRNA functions-mRNA destabilization by perfectly base-paring with mature miR23 a even though there is no perfectly complementary sequence of mature miR-23a in the mammalian genome and such an miRNA molecular mechanism happens in plants. For imaging its target mRNA destabilization regulated by mature miR-23a, gene expression analysis using microarray or differential-display PCR and bioinformatics analysis using a PicTar WEB INTERFACE database (a project of the Rajewsky laboratory at New York University) will be helpful to find miR-23a-downregulated genes directly bound by a mature miR-23a seed sequences or a miRNA response element (MRE). In vivo dual-luciferase imaging of both the CMV/Gluc/ 3xPT_mir23 construct and a reporter construct containing a MRE located in the $3^{\prime}$ UTR of Fluc will provide the information on how miR-23a is generated and how it is functionally acting to destabilize its target mRNA simultaneously. In this investigation, miRNA functional action was not proposed to be visualized during cellular development. We just proposed the proof of concept that the dual-luciferase system might follow the changes of expression or posttranscriptional regulation of a miRNA. In future studies, we will attempt to make this method into a high-throughput one covering all of exclusively expressed miRNAs in a certain specific tissue. Making cell lines persistently express the dual-luciferase system is also a prerequisite to apply this system to follow the longitudinal changes of expression or posttranscriptional regulation of miRNA biogenesis and its mechanistic implications in neuronal development. Notwithstanding these future directions of the mandatory development, we can state that in vivo noninvasive visualization of posttranscriptional miRNA-regulation is now feasible.

The methods currently used to study miRNA expression patterns during neuronal development are Northern blotting, microarrays, and tissue-specific RNA cloning. These modalities are time-consuming, laborious, and inconvenient and, especially, require the sacrifice of animals to monitor miRNA biogenesis. This devised noninvasive imaging methodthough not quantifying the precise amount of pri-miRNA and mature miRNA, - allows us to examine their expression levels simultaneously, and this measurement is repeatable in vivo without sacrificing the animals. Because there is ample reason to believe that the expression patterns of pri-miRNA and mature miRNA represent the posttranscriptional regulation of miRNAs and are related to organ or tissue development $(29,30)$ as well as carcinogenesis $(29,30,32)$, we believe that our technique is useful to probe miRNA biogenesis during neuronal development. Of course, this technique can be applied to follow myogenesis, osteogenesis, and organogenesis in general or other cellular events. The variability in ex vivo monitoring of in vivo cellular events in which animals are sacrificed is minimized by luciferase imaging repeatedly using the same animals with the same grafted cells; thus, the application of molecular imaging to miRNA expression profiling offers a substantial advantage to studies on the dynamics of miRNA sets in vivo. When one considers the difficulty of measuring miRNA expression repeatedly in living animals using traditional techniques, our system is the solution to evaluate the spatial and temporal expression of miRNAs via real-time monitoring and quantitative analysis.

\section{CONCLUSION}

Our dual-luciferase reporter system is proven to be useful to noninvasively monitor the expression and regulation of miRNAs and may provide an understanding of cellular development as well as pathobiology related to various miRNAs in living organisms.

\section{ACKNOWLEDGMENTS}

We are grateful to Dr. V. Narry Kim for her kind donation of miR23P639/Fluc vector. This work was supported by the Nano Bio Regenomics Project and Radiation Brain Science Project of the Korean Science and Engineering Foundation.

\section{REFERENCES}

1. Bartel DP. MicroRNAs: genomics, biogenesis, mechanism, and function. Cell. 2004;116:281-297.

2. Ambros V. The functions of animal microRNAs. Nature. 2004;431:350-355.

3. Reinhart BJ, Slack FJ, Basson M, et al. The 21-nucleotide let-7 RNA regulates developmental timing in Caenorhabditis elegans. Nature. 2000;403:901-906.

4. Dostie J, Mourelatos Z, Yang M, Sharma A, Dreyfuss G. Numerous microRNPs in neuronal cells containing novel microRNAs. RNA. 2003;9:180-186.

5. Xu P, Vernooy SY, Guo M, Hay BA. The Drosophila microRNA Mir-14 suppresses cell death and is required for normal fat metabolism. Curr Biol. 2003;13: 790-795.

6. Brennecke J, Hipfner DR, Stark A, Russell RB, Cohen SM. Bantam encodes a developmentally regulated microRNA that controls cell proliferation and regulates the proapoptotic gene hid in Drosophila. Cell. 2003;113:25-36.

7. Obernosterer G, Leuschner PJ, Alenius M, Martinez J. Post-transcriptional regulation of microRNA expression. RNA. 2006;12:1161-1167.

8. Thomson JM, Newman M, Parker JS, Morin-Kensicki EM, Wright T, Hammond SM. Extensive post-transcriptional regulation of microRNAs and its implications for cancer. Genes Dev. 2006;20:2202-2207.

9. Lee Y, Jeon K, Lee JT, Kim S, Kim VN. MicroRNA maturation: stepwise processing and subcellular localization. EMBO J. 2002;21:4663-4670.

10. Lee Y, Ahn C, Han J, et al. The nuclear RNase III Drosha initiates microRNA processing. Nature. 2003;425:415-419.

11. Lund E, Guttinger S, Calado A, Dahlberg JE, Kutay U. Nuclear export of microRNA precursors. Science. 2004;303:95-98.

12. Yi R, Qin Y, Macara IG, Cullen BR. Exportin-5 mediates the nuclear export of pre-microRNAs and short hairpin RNAs. Genes Dev. 2003;17:3011-3016.

13. Hutvagner G, Zamore PD. A microRNA in a multiple-turnover RNAi enzyme complex. Science. 2002;297:2056-2060.

14. Krichevsky AM, King KS, Donahue CP, Khrapko K, Kosik KS. A microRNA array reveals extensive regulation of microRNAs during brain development. RNA. 2003;9:1274-1281.

15. Smirnova L, Grafe A, Seiler A, Schumacher S, Nitsch R, Wulczyn FG. Regulation of miRNA expression during neural cell specification. Eur J Neurosci. 2005; 21:1469-1477.

16. Miska EA, Alvarez-Saavedra E, Townsend M, et al. Microarray analysis of microRNA expression in the developing mammalian brain. Genome Biol. 2004; 5:R68.1-R68.13

17. Mansfield JH, Harfe BD, Nissen R, et al. MicroRNA-responsive 'sensor' transgenes uncover Hox-like and other developmentally regulated patterns of vertebrate microRNA expression. Nat Genet. 2004;36:1079-1083.

18. Johnson SM, Lin SY, Slack FJ. The time of appearance of the C. elegans let-7 microRNA is transcriptionally controlled utilizing a temporal regulatory element in its promoter. Dev Biol. 2003;259:364-379.

19. Ottobrini L, Ciana P, Biserni A, Lucignani G, Maggi A. Molecular imaging: a new way to study molecular processes in vivo. Mol Cell Endocrinol. 2006; 246:69-75.

20. Doubrovin M, Serganova I, Mayer-Kuckuk P, Ponomarev V, Blasberg RG. Multimodality in vivo molecular-genetic imaging. Bioconjug Chem. 2004;15:1376-1388. 
21. De Wet JR, Wood KV, Helinski DR, DeLuca M. Cloning of firefly luciferase cDNA and the expression of active luciferase in Escherichia coli. Proc Natl Acad Sci USA. 1985;82:7870-7873.

22. Tannous BA, Kim DE, Fernandez JL, Weissleder R, Breakefield XO. Codonoptimized Gaussia luciferase cDNA for mammalian gene expression in culture and in vivo. Mol Ther. 2005;11:435-443.

23. Gould SJ, Subramani S. Firefly luciferase as a tool in molecular and cell biology. Anal Biochem. 1988;175:5-13.

24. Brasier AR, Tate JE, Habener JF. Optimized use of the firefly luciferase assay as a reporter gene in mammalian cell lines. Biotechniques. 1989;7:1116-1122.

25. Lee Y, Kim M, Han J, et al. MicroRNA genes are transcribed by RNA polymerase II. EMBO J. 2004;23:4051-4060.

26. Griffiths-Jones S, Grocock RJ, van Dongen S, Bateman A, Enright AJ. miRBase: microRNA sequences, targets and gene nomenclature. Nucleic Acids Res. 2006; 34:D140-D144.
27. Pachernik J, Bryja V, Esner M, Kubala L, Dvorak P, Hampl A. Neural differentiation of pluripotent mouse embryonal carcinoma cells by retinoic acid: inhibitory effect of serum. Physiol Res. 2005;54:115-122.

28. Ying QL, Stavridis M, Griffiths D, Li M, Smith A. Conversion of embryonic stem cells into neuroectodermal precursors in adherent monoculture. Nat Biotechnol. 2003;21:183-186.

29. Lim LP, Lau NC, Garrett-Engele P, et al. Microarray analysis shows that some microRNAs downregulate large numbers of target mRNAs. Nature. 2005;433:769-773.

30. Wienholds E, Kloosterman WP, Miska E, et al. MicroRNA expression in zebrafish embryonic development. Science. 2005;309:310-311.

31. Song L, Tuan RS. MicroRNAs and cell differentiation in mammalian development. Birth Defects Res C Embryo Today. 2006;78:140-149.

32. Meng F, Henson R, Lang M, et al. Involvement of human micro-RNA in growth and response to chemotherapy in human cholangiocarcinoma cell lines. Gastroenterology. 2006;130:2113-2129. 